\title{
Adaptive sliding mode formation control of multiple underwater robots
}

\author{
BIKRAMADITYA DAS, BIDYADHAR SUBUDHI and BIBHUTI BHUSAN PATI
}

\begin{abstract}
This paper proposes a new adaptive sliding mode control scheme for achieving coordinated motion control of a group of autonomous underwater vehicles with variable added mass. The control law considers the communication constraints in the acoustic medium. A common reference frame for velocity is assigned to a virtual leader dynamically. The performances of the proposed adaptive SMC were compared with that of a passivity based controller. To save the time and traveling distance for reaching the FRP by the follower AUVs, a sliding mode controller is proposed in this paper that drives the state trajectory of the AUV into a switching surface in the state space. It is observed from the obtained results that the proposed SMC provides improved performance in terms of accurately tracking the desired trajectory within less time compared to the passivity based controller. A communication consensus is designed ensuring the transfer of information among the AUVs so that they move collectively as a group. The stability of the overall closed-loop systems are analysed using Lyapunov theory and simulation results confirmed the robustness and efficiency of proposed controller.
\end{abstract}

Key words: AUV, SMC, passivity, cooperative control

\section{Introduction}

An AUV is a submersible undersea vehicle which is equipped with power supply and is controlled by an on board computer and performing an assigned task such as trajectory tracking, path following, navigation etc.. AUVs are compact and low-drag profile crafts powered by underwater DC power thrust [1]. AUVs can be equipped with sensors in order to measure temperature, salinity, pollutant concentration fields, and magnetic vector fields of underwater environment. A team of AUVs in formation is operated for achieving tasks such as mapping, exploration, monitoring of marine environments, data collection for oceanographic missions and autonomous navigation information [2]. Formation Control of AUVs is difficult because of hydrodynamic effects and challenging due to uncertainty in AUV dynamics and communication constraints in the acoustic medium [3].

B. Das and B.B. Pati are with Department of Electrical Engineering, VSS University of Technology, Burla-768018, Odisha, India. B. Subudhi, the corresponding author is with Department of Electrical Engineering, National Institute of Technology, Rourkela -769008, India, e-mail: bidyadharnitrk1@ gmail.com

Received 8.09.2014. Revised 2.11.2014r. 
In underwater communication, acoustic modem has a low data rate, long propagation delay due to the slow speed of sound $(1500 \mathrm{~m} / \mathrm{s})$, the low link quality caused by multipath signal propagation and time variability of the medium. It is quite challenging that design of controller to cover the waypoints between the AUVs in a large uncertain oceanographic region using underwater communication constraints [4]. A topology of information exchange between AUVs is described by a graph theory is very much useful for solving these types of nonlinear unconstrained problems [5]. It is also necessary that all the vehicles in the group to move collectively along a desired path [6]. The control design for trajectory tracking and path following in the presence of modeling parametric uncertainty is a great concern for participating AUVs to put into a formation [7].

Distributed coordination architecture for multi-robot formation control has been proposed in [8]. Nonlinear formation control of marine craft is described by Fossen $e t$ al. [9]. Adaptive design for reference velocity recovery in motion coordination is given in [10]. Arcak et al. proposed passivity as a design tool for group coordination in a synchronized path [11], [12]. Passivity-based techniques and consensus tracking theories are brought together to yield a distributed control strategy proposed by Wang et al. [13]. Sliding mode being a robust controller is chosen to be a suitable candidate for formation control applied with same communication constraints and trajectory. Design of sliding mode controller for a class of second-order under actuated systems is provided by [14]. Trajectory tracking control has been developed and explains the nonlinear horizontal motion dynamics of AUVs [15]. Coordinated path-following control of multiple under actuated autonomous vehicles in the presence of communication failures is proposed by Ghabcheloo et al. [16].

The present work focuses in the development of control algorithm for coordinated motion of a team of AUVs along a desired path in presence of full communications. This objective is achieved by execution control law and following the reference trajectory. A reference trajectory is considered as the dynamic path and the other participating AUVs are assigned to follow the task of the leader using various controller techniques. The motivation behind the formation control owing to the communication constraint i.e. the common velocity signal must be available to all following AUVs that make use of this information. To compare the efficacy of the proposed controller its performances are compared with that of passivity based controller. The stability of the proposed control system is ensured while achieving simultaneous tracking of positions and heading angle based on Lyapunov stability.To develop a SMC with an adaptive mechanism we use Lyapunov stability criteria considering the mass variation of AUV for successful formation control in which coefficient of variable added mass is estimated adaptively [17]. The variable added mass is actually the force and moment on the AUV except the real mass of the moving fluid attached to the AUV. Mass variation means the extra load added or removed from AUV at a particular instant of time while accomplishing the cooperative motion using formation. A communication consensus is used for coordination of AUVs by allocating positions and velocities for the desired trajectories [18]. The objectives of this paper are as follows. 
- Development of an adaptive SMC for co-operative formation of AUVs in presence of variable mass at a certain instant of time based on unchanged communication strategies.

- To compare the performance of the SMC controller having same initial conditions and communication strategies with that of a passivity based controller using various trajectory paths.

The paper is organized as follows. Section 2 gives the modeling of AUV. Section 3 formulates the trajectory tracking problem. Section 4 describes the communication consensus based on tracking control used for formation of AUVs. A detailed description about the design of controller approach is described in Section 5. Section 6 verifies the performance of controller through MATLAB simulation. Section 7 presents the conclusion of this paper.

\section{AUV modeling}

The AUV model in inertial frame can be expressed by the following nonlinear equation [4]

$$
\begin{gathered}
M_{\eta}\left(\eta_{i}\right) \ddot{\eta}_{i}+C_{\eta}\left(\eta_{i}, \dot{\eta}_{i}\right) \dot{\eta}_{i}+D_{\eta}\left(\eta_{i}, \dot{\eta}_{i}\right) \dot{\eta}_{i}+g\left(\eta_{i}\right)=\bar{\tau}_{i} \\
\dot{\eta}_{i}=R\left(\psi_{i}\right) v_{i} .
\end{gathered}
$$

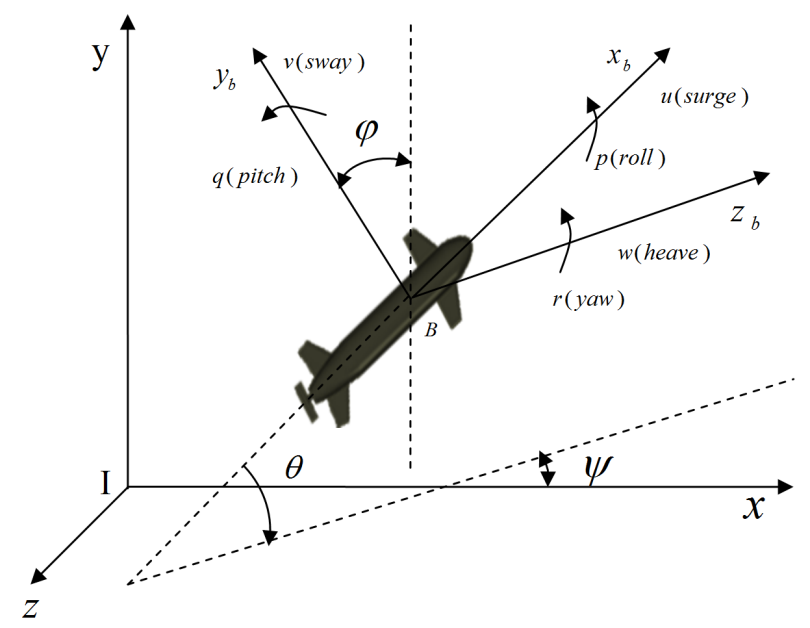

Figure 1: Inertial and body fixed frames of an AUV model.

A schematic representation of an AUV in inertia and body reference frames is shown in Fig. 1 [3]. $M_{\eta}\left(\eta_{i}\right)$ is the inertia matrix including added mass is represented as

$$
M_{\eta}\left(\eta_{i}\right)=R^{T}\left(\psi_{i}\right) M R\left(\psi_{i}\right)
$$


$C_{\eta}\left(\eta_{i}, \dot{\eta}_{i}\right)$ denotes the matrix of Coriolis and centripetal terms including added mass given by

$$
C_{\eta}\left(\eta_{i}, \dot{\eta}_{i}\right)=\left[C(v)-M R^{-1}\left(\psi_{i}\right) \dot{R}\left(\psi_{i}\right)\right] R^{-1}\left(\psi_{i}\right) .
$$

Hydrodynamics damping matrix $D_{\eta}\left(\eta_{i}, \dot{\eta}_{i}\right)$ can be expressed as

$$
D_{\eta}\left(\eta_{i}, \dot{\eta}_{i}\right)=D(v) R^{-1}\left(\psi_{i}\right)
$$

$\eta_{i}=\left[x_{i}, y_{i}, z_{i}, \phi_{i}, \theta_{i}, \psi_{i}\right]^{T}$ describes the position and orientation of the vehicle with respect to the inertial or earth fixed reference frame. $v_{i}=\left[u_{i}, v_{i}, w_{i}, p_{i}, q_{i}, r_{i}\right]^{T}$ is the translational and rotational velocities of the vehicle with respect to the body-fixed reference frame. $\bar{\tau}_{i}=\left[X_{i}, Y_{i}, Z_{i}, K_{i}, M_{i}, N_{i}\right]^{T}$ is the control input vector in horizontal plane. $g\left(\eta_{i}\right)$ denotes the vector of gravitational forces and moments, $\bar{\tau}_{i}$ is the vector of force and moments acting on the AUV in the body fixed frame. $\bar{\tau}_{i}$ is given by

$$
\bar{\tau}_{i}=R\left(\psi_{i}\right) \tau_{i}
$$

$R\left(\psi_{i}\right)$ denotes the velocity transformation matrix between vehicle and earth fixed frame. The rotation matrix of the AUV can be expressed as

$$
R\left(\psi_{i}\right)=\left[\begin{array}{ccc}
\cos \left(\psi_{i}\right) & -\sin \left(\psi_{i}\right) & 0 \\
\sin \left(\psi_{i}\right) & \cos \left(\psi_{i}\right) & 0 \\
0 & 0 & 1
\end{array}\right]
$$

Assumption 3 Referring to equations (3-5), we assume the following properties in the AUV dynamics [2]

- $M_{\mathfrak{\eta}}\left(\eta_{i}\right)$ is symmetric positive stable for any number of AUV, $i=1,2, \ldots, N$

$$
\lambda_{\min }\left(M_{i}\right)\|x\|_{2}^{2} \leqslant x^{T} M_{\eta}\left(\eta_{i}\right) x \leqslant \lambda_{\max }\left(M_{i}\right)\|x\|_{2}^{2}, \forall x \neq 0 .
$$

- $\dot{M}_{\eta}\left(\eta_{i}\right)-2 C_{\eta}\left(\eta_{i}, \dot{\eta}_{i}\right)$ is a skew $e_{i} \rightarrow 0$ symmetric and which is can be termed as

$$
x^{T}\left(\frac{1}{2} \dot{M}_{\eta}\left(\eta_{i}\right)-2 C_{\eta}\left(\eta_{i}, \dot{\eta}_{i}\right)\right) x=0, \quad \forall x \in \mathfrak{R}^{3} .
$$

- The damping matrix $D_{\eta}\left(\eta_{i}, \dot{\eta}_{i}\right)$ is positive such that

$$
x^{T} D_{\eta}\left(\eta_{i}, \dot{\eta}_{i}\right) x>0, \quad \forall x \neq 0 .
$$

- Embedding a load will not only change the mass matrix, but it will also induce torques as the gravity and buoyancy centers will not coincide anymore and hence $g$ will change. But as it is considered for three DOF of motion along $(x, y)$ axis, hence $g\left(\eta_{i}\right)=0$.

- Centre of mass and centre of buoyancy coincide with each other and other terms such as roll motions and hydrodynamic terms of higher order are assumed to be negligible. 


\section{Problem statement}

Consider $N$ number of similar AUVs deployed for a task to be achieved through a cooperative motion plan $i=1,2, \ldots, N$. Let the AUVs accomplish the cooperative motion using formation. We choose a Leader-Follower formation topology in which one of the AUV is chosen as the leader and the rest are considered as the follower. It is assumed that the common velocity and position signals must be available to all the AUVs in all above mentioned references. Formation Control of single networked AUVs is difficult and challenging due to uncertainty in its dynamics and communication constraints in the acoustic medium. This problem can be overcome by introducing communication consensus for both formation and tracking as shown in Fig. 2 [13].

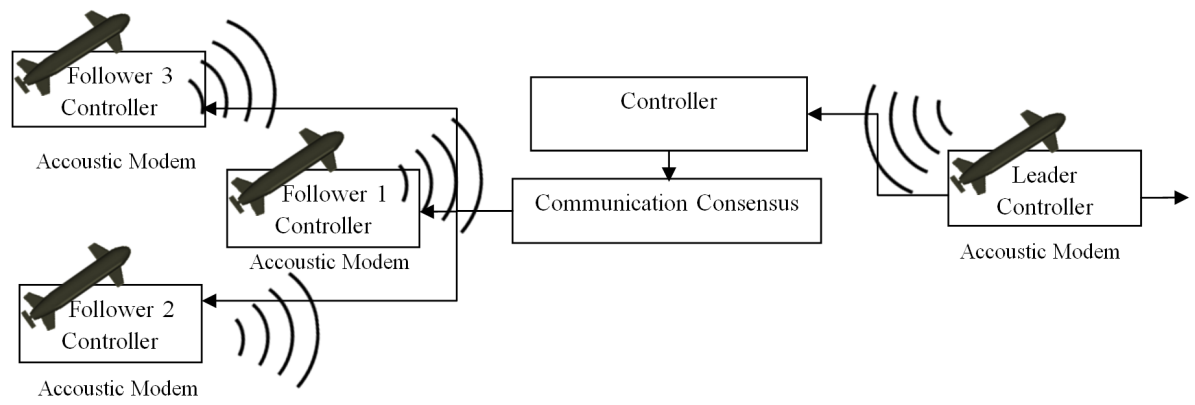

Figure 2: Formation Control of multiple AUVs using communication constraints.

The problem statement is as follows. The error surface for each AUV is used to provide its dynamics from a feedback signal to the error vector $\dot{\eta}_{0, i}-v_{0, i}(t)$ for passivity based controller [13]. The proposed SMC applied to the systematic control design having switching surface $\dot{\eta}_{i}\left(\theta_{i}\right)-\dot{\eta}_{r}\left(\theta_{i}\right)$ in the state space by maintaining the trajectory on the same surface with and without variable added mass. All follower AUVs grasping a mass variation at a certain instant of time will be in the desired formation and consensus with the virtual leader, when the position vector of each AUV is synchronized. The neighbor AUVs $i$ and $j$ exchange their information through a common communication consensus algorithm by accessing the synchronization error $\theta_{i}-\theta_{j}$. The formulation for the AUVs in desired formation configuration in two dimensional space is

$$
\begin{gathered}
\lim _{t \rightarrow \infty}\left|\dot{\eta}_{0, i}-v_{0, i}\left(\theta_{i}\right)\right|=0, \quad \forall i \in(1,2, \ldots, N) \\
\lim _{t \rightarrow \infty}\left|\dot{\eta}_{i}\left(\theta_{i}\right)-\dot{\eta}_{r}\left(\theta_{i}\right)\right|=0, \quad \forall i \in(1,2, \ldots, N) \\
\lim _{t \rightarrow \infty}\left|\theta_{i}-\theta_{j}\right|=0, \quad \forall i \in(1,2, \ldots, N)
\end{gathered}
$$

where $\eta_{r}$ represents the reference path for the group of AUVs and $v_{0, i}$ is the velocity of the $i$ th vehicle for tracking the desired leader path. $\theta_{i}$ is the consensus tracking algorithm as discussed in section 4 . 


\section{Communication consensus}

A bidirectional information flow exist between the leader and follower AUVs described by graph theory . Here all followers track the virtual leader in the absence of velocity measurements. The synchronization error can be exchanged between the AUVs, considering one of the vertices to be the positive end of the edge $k$. So the incidence matrix $D(g)$ is defined

$$
p_{i k}= \begin{cases}+1 & \text { if } i \text { th AUV is the positive end of } k \\ -1 & \text { if } i \text { th AUV is the negative end of } k\end{cases}
$$

The associated adjacency matrix associated with graph theory is $A=\left[a_{i j}\right] \in R^{N \times N}$. Two members, $i$ and $j$ are neighbor AUVs if they can access the synchronization error $\theta_{i}-\theta_{j}$. Consensus tracking means consensus or full communication with the virtual leader, the details has been described by Cao et.al [22]. The objective of consensus tracking is that a group of followers tracks a dynamic leader with exchange of information. The objective of $\theta_{i}$ is to track the leader in presence of consensus tracking represented as,

$$
\lim _{t \rightarrow \infty}\left|\theta_{i}-\theta_{j}\right|=0, \quad \forall i, j \in(1,2, \ldots, N)
$$

So the distributed consensus tracking algorithm for $\dot{\theta}_{i}$ may be defined as [22]

$$
\dot{\theta}_{i}=-\gamma \sum_{j \in \overline{N_{i}}(t)} a_{i j}\left(\theta_{i}-\theta_{j}\right)-\beta_{c} \operatorname{sgn}\left[\sum_{j \in \overline{N_{i}}(t)} a_{i j}\left(\theta_{i}-\theta_{j}\right)\right]
$$

$\bar{N}_{i} \subseteq\{0,1, \ldots, n\}$ denotes the neighbor set of follower $i$ in the team consisting of the $n$ followers and the virtual leader. Considering the case of a switching network topology by assuming that $j \in \overline{N_{i}(t)}, i=1,2, \ldots, n, j=0,1, \ldots, n$, if $\left|\theta_{i}-\theta_{j}\right|<R$ at time $t$ and $j \notin \overline{N_{i}(t)}$, where $R$ denotes the communication sensing radius of the AUV [21]. $a_{i j}$, $i=1,2, \ldots, n, j=0,1, \ldots, n$, are positive constants, $\gamma$ is a nonnegative constant, $\beta_{c}$ is a positive constant, and $\operatorname{sgn}[\cdot]$ is the signum function. As undirected graph is connected, so at least one value of $a_{i 0}$ is nonzero. $a_{i 0}$ is a positive constant if the virtual leader's position is available to follower $i$. Here $\theta_{0}$ is updated to force the speed along the reference trajectory path, $\dot{\theta}_{0}$ to follow a speed assignment $v_{0}$ assuming $\dot{\theta}_{0}<u$, and $u$ is a positive constant. Then the Lyapunov candidate function is given by $V$ may not be smooth but regular. Here differential inclusions and non-smooth analysis is used to analyze the stability [22].

$$
\dot{\theta}_{i} \in \in^{\text {a.e. }}-K\left[\gamma \sum_{j \in \overline{N_{i}(t)}} a_{i j}\left(\theta_{i}-\theta_{j}\right)+\beta_{c} s g n\left[\sum_{j \in \bar{N}_{i}(t)} a_{i j}\left(\theta_{i}-\theta_{j}\right)\right]\right]
$$


where a.e. denotes "almost everywhere." and $K[\cdot]$ is the differential inclusion [22] and the generalized derivative of $V$ is given by

$$
V \leqslant-\gamma \widetilde{\theta}^{T}[\hat{\chi}(t)]^{2} \widetilde{\theta}-\left(\beta_{c}-u\right)\|\hat{\chi}(t) \widetilde{\theta}\|
$$

$\widetilde{\theta}$ is the column stack vector of $\widetilde{\theta}_{i}, i=1,2, \ldots, n$, with $\widetilde{\theta}_{i}=\theta_{i}-\theta_{0}, \hat{\chi}(t)=\left[\hat{m}_{i j}(t)\right] \in \mathfrak{R}^{n \times n}$ is defined as [22]

$$
\hat{m}_{i j}(t)= \begin{cases}-a_{i j,}, & j \in \bar{N}_{i}(t), j \neq i \\ 0, & j \notin \bar{N}_{i}(t), j \neq i \\ \sum_{k \in \bar{N}_{i}(t)} a_{i k}, & j=i\end{cases}
$$

$\hat{\chi}(t)$ is symmetric positive definite at each time instant under the condition of the theorem. Because $\beta_{c}>u$, it then follows that the generalized derivative of $V$ is negative definite and $\|\widetilde{\theta}(t)\| \rightarrow 0$ as $t \rightarrow \infty$ [22]. Therefore, we can get that $\theta_{i}(t) \rightarrow \theta_{0}(t)$ as $t \rightarrow \infty$.

\section{Controller design}

In this section, systematic design procedures for achieving solution to control objectives for formation control are formulated with the help of communication consensus as described in the previous section.

\subsection{Passively based controller}

In order to compare the effectiveness of the proposed adaptive SMC controller, we first design a passivity based controller [13] for achieving formation of a group of AUVs. The structure of the passivity controller is as shown in Fig. 3. Each AUV is intended to follow the following trajectory given by [13]

$$
\eta_{d, i}\left(\theta_{i}\right)=\eta_{0, i}\left(\theta_{i}\right)+R\left(\left(\psi_{i}\left(\theta_{i}\right)\right) l_{i}\right.
$$

Differentiating both sides of (20) with respect to time gives

$$
\begin{aligned}
& \dot{\eta}_{d, i}\left(\theta_{i}\right)=\dot{\eta}_{0, i}\left(\theta_{i}\right)+\dot{f}_{i} \\
& \ddot{\eta}_{d, i}\left(\theta_{i}\right)=\ddot{\eta}_{0, i}\left(\theta_{i}\right)+\ddot{f}_{i}
\end{aligned}
$$

where the desired path for $i$ th AUV is given by $\eta_{d, i}\left(\theta_{i}\right)=\left[x_{d, i}\left(\theta_{i}\right), y_{d, i}\left(\theta_{i}\right), \psi_{d, i}\left(\theta_{i}\right)\right]^{T}$. A formation reference point (FRP) is designed with a set of designation vectors $f_{i}=R\left(\left(\psi_{i}\left(\theta_{i}\right)\right) l_{i}, l_{i} \in \mathfrak{R}^{3}\right.$ for $i$ th AUV, $i=1,2, \ldots, N$. We define the $\eta_{0, i}\left(\theta_{i}\right)=$ $\left[x_{0, i}\left(\theta_{i}\right), y_{0, i}\left(\theta_{i}\right), \psi_{0, i}\left(\theta_{i}\right)\right]^{T}$ as the FRP for $i$ th AUV. $R\left(\psi\left(\theta_{i}\right)\right)$ is the rotation matrix, is 


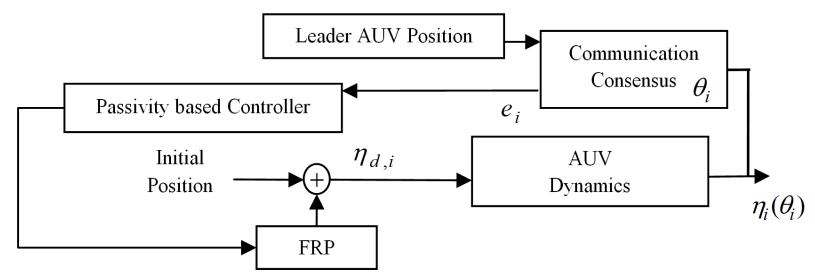

Figure 3: Formation control using passivity based controller.

the angle of tangent vector in the inertial frame and $\theta_{i}$ is the communication tracking algorithm as described in Section 4.

The desired heading $\psi_{i}\left(\theta_{i}\right)$ can be calculated as the angle of the tangent vector in the inertial frame.

$$
\psi_{i}\left(\theta_{i}\right)=\arctan \left(\frac{y_{0, i}^{\prime}\left(\theta_{i}\right)}{x_{0, i}^{\prime}\left(\theta_{i}\right)}\right)
$$

where $x_{0, i}^{\prime}\left(\theta_{i}\right)$ and $y_{0, i}^{\prime}\left(\theta_{i}\right)$ represent the partial derivative of $x_{0, i}\left(\theta_{i}\right)$ and $y_{0, i}\left(\theta_{i}\right)$ respectively.

$$
R\left(\psi_{i}\left(\theta_{i}\right)\right)=\left[\begin{array}{ccc}
\cos \left(\psi_{i}\left(\theta_{i}\right)\right) & -\sin \left(\psi_{i}\left(\theta_{i}\right)\right) & 0 \\
\sin \left(\psi_{i}\left(\theta_{i}\right)\right) & \cos \left(\psi_{i}\left(\theta_{i}\right)\right) & 0 \\
0 & 0 & 1
\end{array}\right] .
$$

The idea behind the passivity based design is to derive an internal feedback control law for each AUV to provide its dynamics from an external feedback signal $\alpha_{i}$ to the error vector $\left(\dot{\eta}_{0, i}-v_{0, i}\right)$, where $v_{0, i}$ is the velocity of the AUV [13]. This means

$$
\begin{gathered}
\lim _{t \rightarrow \infty}\left|\dot{\eta}_{0, i}-v_{0, i}(t)\right|=0, \quad \forall i \in(1,2, \ldots, N) \\
\alpha_{i}=-\sum_{k=1}^{p} l_{i k} \varphi_{k}\left(z_{k}\right), \quad \forall i \in(1,2, \ldots, N)
\end{gathered}
$$

where $z_{k}$ is the coordination error between $i$ th and $j$ th AUV connected by communication link $l_{i k}$ at each node $k$ and $\phi_{k}(\cdot)$ is the feed forward non-negative non-linear function as described in the section 4 . The error vector is defined as

$$
e_{i}=\dot{\eta}_{0, i}-v_{0, i}
$$

Differentiating (27) gives

$$
\ddot{\eta}_{0, i}=\dot{e}_{i}+\dot{v}_{0, i} .
$$

Substituting the expression for $\ddot{\eta}_{d, i}\left(\theta_{i}\right)$ from (22) in equation (1) one can obtain

$$
M_{\eta}\left(\eta_{i}\right)\left(\ddot{\eta}_{0, i}\left(\theta_{i}\right)+\ddot{f}_{i}\right)+C_{\eta}\left(\eta_{i}, \dot{\eta}_{i}\right)\left(\dot{\eta}_{0, i}\left(\theta_{i}\right)+\dot{f}_{i}\right)+D_{\eta}\left(\eta_{i}, \dot{\eta}_{i}\right)\left(\dot{\eta}_{0, i}\left(\theta_{i}\right)+\dot{f}_{i}\right)+g\left(\eta_{i}\right)=\bar{\tau}_{i} .
$$


Substituting (28) in (29), we have

$M_{\eta}\left(\eta_{i}\right)\left(\dot{e}_{i}+\dot{v}_{0, i}+\ddot{f}_{i}\right)+C_{\eta}\left(\eta_{i}, \dot{\eta}_{i}\right)\left(e_{i}+v_{0, i}+\dot{f}_{i}\right)+D_{\eta}\left(\eta_{i}, \dot{\eta}_{i}\right)\left(e_{i}+v_{0, i}+\dot{f}_{i}\right)+g\left(\eta_{i}\right)=\bar{\tau}_{i}$.

The control input $\bar{\tau}_{i}$ can be generated so that the error $e_{i}$ converges to zero.

$\bar{\tau}_{i}=\left(C_{\eta}\left(\eta_{i}, \dot{\eta}_{i}\right)+D_{\eta}\left(\eta_{i}, \dot{\eta}_{i}\right)\right)\left(v_{0, i}+\dot{f}_{i}\right)+M_{\eta}\left(\eta_{i}\right)\left(\dot{v}_{0, i}+\ddot{f}_{i}\right)+g\left(\eta_{i}\right)-K_{d i}\left(\dot{\eta}_{0, i}-v_{0, i}\right)+\alpha_{i}$.

$K_{d i}$ is the positive control gain matrix. The positive definite used in passivity control are radially unbounded storage function [13]. The positive definiteness of passivity based control approach is given as

$\bar{\tau}_{i}=\left(C_{\eta}\left(\eta_{i}, \dot{\eta}_{i}\right)+D_{\eta}\left(\eta_{i}, \dot{\eta}_{i}\right)\right)\left(v_{0, i}+\dot{f}_{i}\right)+M_{\eta}\left(\eta_{i}\right)\left(\dot{v}_{0, i}+\ddot{f}_{i}\right)+g\left(\eta_{i}\right)-K_{d i}\left(\dot{\eta}_{0, i}-v_{0, i}\right)+\alpha_{i}$.

So according to the definition of passivity based control [11] and [14], it can be observed that passivity can be achieved through $\alpha_{i}$ and $e_{i}$. This means that the follower AUVs will have to reach the FRP to follow the trajectory which is very much time consuming.

\subsection{Sliding mode based formation control}

The control objective is to find a nonlinear control law that makes the AUV asymptotically tracking a desired trajectory. SMC method applied to the systematic control design for the reduction of the system order. The measure of tracking through SMC is represented as [15], as shown in Fig. 4 [17]

$$
s=\dot{\widetilde{\eta}}_{i}+\Upsilon \widetilde{\eta}_{i}
$$

where $\eta_{i}\left(\theta_{i}\right)=\left[x_{i}\left(\theta_{i}\right), y_{i}\left(\theta_{i}\right), \psi_{i}\left(\theta_{i}\right)\right]^{T}$ for $i$ th AUV, $i=1,2, \ldots, N . \widetilde{\eta}_{i}\left(\theta_{i}\right)=\eta_{i}\left(\theta_{i}\right)-\eta_{d}$ is the earth fixed reference error, $\Upsilon_{l}$ is a diagonal positive design matrix. The virtual reference trajectory in Earth-fixed coordinates are defined as

$$
\dot{\eta}_{r}\left(\theta_{i}\right)=\dot{\eta}_{d}-\Upsilon_{l} \widetilde{\eta}_{i}\left(\theta_{i}\right)
$$

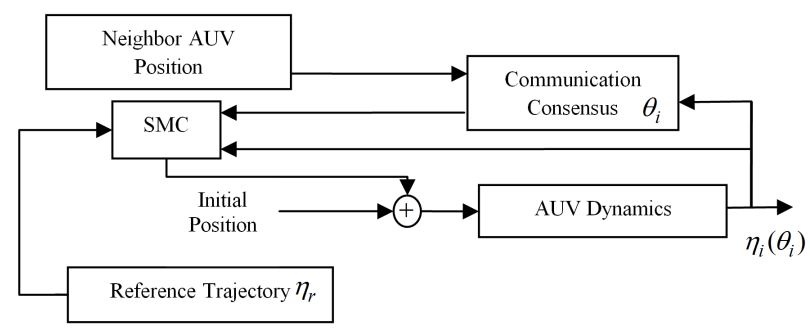

Figure 4: Formation control using SMC based controller.

Defining sliding surface $s$ as

$$
s=\dot{\widetilde{\eta}}_{i}\left(\theta_{i}\right)+\Upsilon_{l} \widetilde{\eta}_{i}\left(\theta_{i}\right)=\dot{\eta}_{i}\left(\theta_{i}\right)-\dot{\eta}_{r}\left(\theta_{i}\right)
$$


Equation (35) can be rewritten as

$$
\ddot{\eta}_{i}\left(\theta_{i}\right)=\dot{s}+\ddot{\eta}_{r}\left(\theta_{i}\right) .
$$

It is assumed that the reference trajectory $\eta_{r}$ is smooth and bounded by $\eta_{d}, \dot{\eta}_{d}, \ddot{\eta}_{d}$. Substituting the expression for $\ddot{\eta}_{i}\left(\theta_{i}\right)$ from (36) in equation (1), we have

$$
\begin{aligned}
& M_{\eta}\left(\eta_{i}\right) \dot{s}=-\left(C_{\eta}\left(\eta_{i}, \dot{\eta}_{i}\right)+D_{\eta}\left(\eta_{i}, \dot{\eta}_{i}\right)\right) s-M_{\eta}\left(\eta_{i}\right) \ddot{\eta}_{r}\left(\theta_{i}\right)-C_{\eta}\left(\eta_{i}, \dot{\eta}_{i}\right) \dot{\eta}_{r}\left(\theta_{i}\right) \\
& -D_{\eta}\left(\eta_{i}, \dot{\eta}_{i}\right) \dot{\eta}_{r}\left(\theta_{i}\right)+\tau_{i}-g\left(\eta_{i}\right) .
\end{aligned}
$$

Equation (37) can be rewritten as

$$
M_{\eta}\left(\eta_{i}\right) \dot{s}=-C_{\eta}\left(\eta_{i}, \dot{\eta}_{i}\right) s-D_{\eta}\left(\eta_{i}, \dot{\eta}_{i}\right) s+\left[\tau_{i}-M \dot{v}_{r}\left(\theta_{i}\right)-C(v) v_{r}\left(\theta_{i}\right)-D_{\eta}(v) v_{r}\left(\theta_{i}\right)-g\left(\eta_{i}\right)\right]
$$

where $v_{r}\left(\theta_{i}\right)=\dot{\eta}_{r}\left(\theta_{i}\right) R^{-1}\left(\psi_{i}\left(\theta_{i}\right)\right)$. Let $\dot{s}=0$

$$
\tau_{e q v}=M \dot{v}_{r}\left(\theta_{i}\right)+C(v) v_{r}\left(\theta_{i}\right)+D(v) v_{r}\left(\theta_{i}\right)+g\left(\eta_{i}\right)+C_{\eta}\left(\eta_{i}, \dot{\eta}_{i}\right) s+D_{\eta}\left(\eta_{i}, \dot{\eta}_{i}\right) s .
$$

Total control input is given by

$$
\tau=\tau_{e q v}+\tau_{s w} .
$$

$\tau_{s w}$ is the switching control part of SMC input. Using SMC $s \rightarrow 0$ in finite time and also with desired convergence rate, the dynamics of the sliding mode is required to have the following form.

$$
\dot{s}=w s-K \operatorname{sgn}(s)
$$

where $w \in \operatorname{diag}\left(w_{i}\right), K \in \operatorname{diag}\left(k_{i}\right), w_{i}>0, k_{i}>0$ for $i \in(1,2, \ldots, N)$. So substituting (41) in (38) we have

$$
\begin{aligned}
& \tau_{s w}=-M_{\eta}\left(\eta_{i}\right)(w s+K \operatorname{sgn}(s)) \\
& \tau=M \dot{v}_{r}\left(\theta_{i}\right)+C(v) v_{r}\left(\theta_{i}\right)+D(v) v_{r}\left(\theta_{i}\right)+g\left(\eta_{i}\right)+C_{\eta}\left(\eta_{i}, \dot{\eta}_{i}\right) s+D_{\eta}\left(\eta_{i}, \dot{\eta}_{i}\right) s \\
& -M_{\eta}\left(\eta_{i}\right)(w s+K \operatorname{sgn}(s)) .
\end{aligned}
$$

So the control law for SMC is given by (43).The control law of SMC given in (43) is uniformly globally stable [15]. Considering the Lyapunov candidate function as [19]

$$
\dot{V}=-s^{T} w s-s^{T} K \operatorname{sgn}(s) \leqslant 0
$$

where $w$ and $K$ are positive matrix and chosen such that it avoids anti-jamming and high frequency chattering. $M$ is a positive matrix, $M_{\eta}\left(\eta_{i}\right)$ is invertible. So the control for the SMC is stable if and only if, it satisfies

$$
\dot{s}_{i}=-w_{i} s-K_{i} \operatorname{sgn}\left(s_{i}\right), \quad s_{i} \in s \text { and } s_{i}\left(t_{0}\right) \neq 0 .
$$

Solution of the above equation satisfying the condition $s_{i}(t)=0$ is

$$
s_{i}(t)=\left[\left|s_{i}\left(t_{0}\right)\right|+w_{i}^{-1} k_{i}\right] e^{-w_{i}\left(t-t_{0}\right)}-w_{i}^{-1} k_{i} .
$$

Sliding model will be reached at time period

$$
t \geqslant t_{0}-\ln \left[\frac{k_{i} /\left(k_{i}+w_{i}\left|s_{i}\left(t_{0}\right)\right|\right)}{w_{i}}\right] .
$$




\subsection{Adaptive sliding mode controller}

It is difficult to obtain the position, velocity and to compute the control input for cooperative formation of AUVs grasping payload mass variation. An adaptive SMC may be designed to hold the system trajectory on the sliding surface and the chattering-free implementation as shown in Fig. 5. The variable added load (variable mass) to the mass matrix of AUV is

$$
\widetilde{M}=M+\left|\delta_{M}\right|
$$

where $\widetilde{M}$ denotes the modified global mass matrix and $\left|\delta_{M}\right|$ is the added mass matrix at a certain instant of time to AUV.

$$
\left|\delta_{M}\right|=\left[\begin{array}{ccc}
\delta_{M_{11}} & 0 & 0 \\
0 & \delta_{M_{22}} & 0 \\
0 & 0 & \delta_{M_{33}}
\end{array}\right]
$$

The modified inertia matrix is given as

$$
\widetilde{M}_{\eta}\left(\eta_{i}\right)=\widetilde{M} R^{-1}\left(\psi_{i}\right)=\left(M+\left|\delta_{M}\right|\right) R^{-1}\left(\psi_{i}\right)
$$

The modified Coriolis matrix is given as

$$
\widetilde{C}_{\eta}\left(\eta_{i}, \dot{\eta}_{i}\right)=C_{\eta}\left(\eta_{i}, \dot{\eta}_{i}\right)-P L
$$

where $P L=\left|\delta_{M}\right| R^{-1}\left(\psi_{i}\right) \dot{R}\left(\psi_{i}\right) R^{-1}\left(\psi_{i}\right)$. The hydrodynamics damping and lift matrix will be unaffected by variable added mass matrix and can be expressed as (10). To move the desired path by the follower with the effect of variable added mass after a certain instant of time

$$
\widetilde{M}_{\eta}\left(\eta_{i}\right) \ddot{\eta}_{i}+\widetilde{C}_{\eta}\left(\eta_{i}, \dot{\eta}_{i}\right) \dot{\eta}_{i}+D_{\eta}\left(\eta_{i}, \dot{\eta}_{i}\right) \dot{\eta}_{i}+g\left(\eta_{i}\right)=\tau_{i}
$$

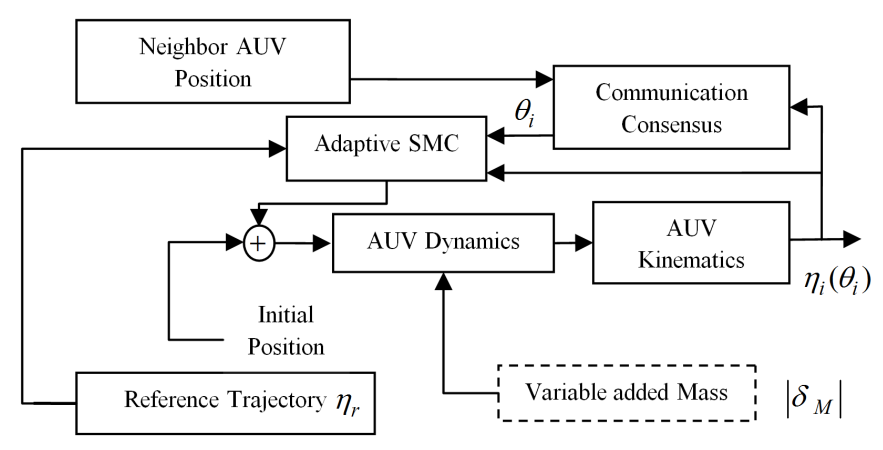

Figure 5: Formation control using adaptive SMC based controller with variable added mass. 
Substituting the expression for $\ddot{\eta}_{i}\left(\theta_{i}\right)$ from (36) in equation (52), we have

$$
\widetilde{M}_{\eta}\left(\eta_{i}\right)\left(\dot{s}+\ddot{\eta}_{r}\left(\theta_{i}\right)\right)+\widetilde{C}_{\eta}\left(\eta_{i}, \dot{\eta}_{i}\right)\left(s+\dot{\eta}_{r}\left(\theta_{i}\right)\right)+D_{\eta}\left(\eta_{i}, \dot{\eta}_{i}\right)\left(s+\dot{\eta}_{r}\left(\theta_{i}\right)\right)+g\left(\eta_{i}\right)=\tau_{i}
$$

The control input $\tau_{i}$ is designed as follows in order to follow the reference on setting $\dot{s}=0$

$$
\begin{aligned}
& -\left[C_{\eta}\left(\eta_{i}, \dot{\eta}_{i}\right) s+D_{\eta}\left(\eta_{i}, \dot{\eta}_{i}\right) s\right]+P L s-\left|\delta_{M}\right| R^{-1}\left(\psi_{i}\right) \ddot{\eta}_{r}\left(\theta_{i}\right)+P L \dot{\eta}_{r}\left(\theta_{i}\right) \\
& \quad-\left[M_{\eta}\left(\eta_{i}\right) \ddot{\eta}_{r}\left(\theta_{i}\right)+C_{\eta}\left(\eta_{i}, \dot{\eta}_{i}\right) \dot{\eta}_{r}\left(\theta_{i}\right)+D_{\eta}\left(\eta_{i}, \dot{\eta}_{i}\right) \dot{\eta}_{r}\left(\theta_{i}\right)+g\left(\eta_{i}\right)\right]+\tau_{i}=0 .
\end{aligned}
$$

For body fixed reference, (54) can be rewritten as

$$
\begin{aligned}
& \tau_{i}-\left|\delta_{M}\right| R^{-1}\left(\psi_{i}\right) \ddot{\eta}_{r}\left(\theta_{i}\right)+P L \dot{\eta}_{r}\left(\theta_{i}\right)+P L s=M \dot{v}_{r}+C(v) v_{r}+D(v) v_{r}+g\left(\eta_{i}\right) \\
& +\left[C_{\eta}\left(\eta_{i}, \dot{\eta}_{i}\right) s+D_{\eta}\left(\eta_{i}, \dot{\eta}_{i}\right) s\right] .
\end{aligned}
$$

To guarantee that sliding mode s tends to zero in finite time and also with desired convergence rate, the dynamics of the sliding mode is required to have the following form [17]

$$
\dot{s}=w s-K \text { sat }\left(\frac{s}{\phi}\right)
$$

$\varphi>0$ is the width of the boundary and sat $\left(\frac{s}{\phi}\right)$ is defined as

$$
\operatorname{sat}\left(\frac{s}{\varphi}\right)=\left\{\begin{array}{c}
1 \\
\operatorname{sgn}\left(\frac{s}{\varphi}\right)
\end{array} \mid \begin{array}{l}
\frac{s}{\varphi} \mid \leqslant 1 \\
\frac{s}{\varphi} \mid \geqslant 1
\end{array}\right.
$$

$K$ is the reaching condition for the sliding mode. The chattering phenomenon can be minimized by replacing the discontinuous control law term $\operatorname{sgn}(s)$ as $\operatorname{sat}(s)$ function around the switching surface [17].

$$
\tau_{s w}=-\tilde{M}\left(\eta_{i}\right)\left[w s+K \operatorname{sat}\left(\frac{s}{\varphi}\right)\right] .
$$

Thus, after plugging in (58) into (55), one can obtain

$$
\begin{aligned}
& \tau_{i}-\left|\delta_{M}\right| R^{-1}\left(\psi_{i}\right) \ddot{\eta}_{r}\left(\theta_{i}\right)+\left|\delta_{M}\right|\left[w s+K s a t\left(\frac{s}{\varphi}\right)\right]+P L \dot{\eta}_{r}\left(\theta_{i}\right)+P L s \\
& \quad=\left[M \dot{v}_{r}+C(v) v_{r}+D(v) v_{r}+g\left(\eta_{i}\right)-M\left[w s+K \operatorname{sat}\left(\frac{s}{\varphi}\right)\right]\right]+\left[C_{\eta}\left(\eta_{i}, \dot{\eta}_{i}\right) s+D_{\eta}\left(\eta_{i}, \dot{\eta}_{i}\right) s\right] .
\end{aligned}
$$

As it is considered as for three DOF of motion along $(x, y)$ axis, hence it can be assumed that $g\left(\eta_{i}\right)=0$. 
Theorem 1 The control law (59) of an adaptive SMC must satisfy the Lyapunov stability criteria and tuning law $\dot{\hat{\delta}}$ is globally uniformly ultimately bounded [17].

Assumption 2 The dynamics equation (52) of AUV is affected by variable mass provides the following properties [2]. For any number of $i \in(1,2, \ldots, N)$ AUV

$$
\begin{gathered}
\lambda_{\min }\left(\widetilde{M}_{i}\right)\|x\|_{2}^{2} \leqslant x^{T} \widetilde{M}_{\eta}\left(\eta_{i}\right) x \leqslant \lambda_{\max }\left(\widetilde{M}_{i}\right)\|x\|_{2}^{2}, \forall x \neq 0 \\
x^{T}\left(\frac{1}{2} \dot{\widetilde{M}}_{\eta}\left(\eta_{i}\right)-2 \widetilde{C}_{\eta}\left(\eta_{i}, \dot{\eta}_{i}\right)\right) x=0, \quad \forall x \in \mathfrak{R}^{3} .
\end{gathered}
$$

We can define the Lyapunov candidate function as

$$
\begin{aligned}
& \ddot{\eta}_{i}=-\tilde{M}_{\eta}^{-1}\left(\eta_{i}\right)\left(C_{\eta}\left(\eta_{i}, \dot{\eta}_{i}\right)+D_{\eta}\left(\eta_{i}, \dot{\eta}_{i}\right)\right) \dot{\eta}_{i} \\
& +\widetilde{M}_{\eta}^{-1}\left(\eta_{i}\right)\left(\left|\delta_{M}\right| R^{-1}\left(\psi_{i}\right) \dot{R}\left(\psi_{i}\right) R^{-1}\left(\psi_{i}\right)\right) \dot{\eta}_{i}+\tilde{M}_{\eta}^{-1}\left(\eta_{i}\right) \tau
\end{aligned}
$$

Define $b=\widetilde{M}_{\eta}^{-1}\left(\eta_{i}\right)$ and $\left|\delta_{M}\right|=\delta$

$$
\ddot{\eta}_{i}=f\left(\eta_{i}, \dot{\eta}_{i}\right)+\delta h\left(\eta_{i}, \dot{\eta}_{i}\right)+b \tau
$$

where

$$
\begin{gathered}
f\left(\eta_{i}, \dot{\eta}_{i}\right)=-\widetilde{M}_{\eta}^{-1}\left(\eta_{i}\right)\left(C_{\eta}\left(\eta_{i}, \dot{\eta}_{i}\right)+D_{\eta}\left(\eta_{i}, \dot{\eta}_{i}\right)\right) \dot{\eta}_{i} \\
h\left(\eta_{i}, \dot{\eta}_{i}\right)=\widetilde{M}_{\eta}^{-1}\left(\eta_{i}\right)\left(R^{-1}\left(\psi_{i}\right) \dot{R}\left(\psi_{i}\right) R^{-1}\left(\psi_{i}\right)\right) \dot{\eta}_{i}
\end{gathered}
$$

On setting $\dot{s}=0$ and adding control term we have final adaptive sliding control law

$$
\tau=\hat{b}^{-1}\left[-\hat{f}\left(\eta_{i}, \dot{\eta}_{i}\right)-\hat{\delta} \hat{h}\left(\eta_{i}, \dot{\eta}_{i}\right)+\ddot{\eta}_{r}-K s a t(s)\right]
$$

We choose a Lyapunov candidate function as

$$
V(t)=\frac{1}{2} s^{2}+\frac{1}{2} \gamma(\boldsymbol{\delta}-\hat{\boldsymbol{\delta}})^{2}
$$

where $\gamma \in \Re$ represents positive adaptive gain constant and $\dot{\delta}=0$ since $\left|\delta_{M}\right|$ is a constant mass parameter.

$$
\dot{V}(t)=s \dot{s}-\gamma \widetilde{\delta} \dot{\hat{\delta}}
$$

where $\widetilde{\delta}=\delta-\hat{\delta}, s \cdot \operatorname{sgn}(s)=|s|$ and putting the value of $\dot{s}$

$$
\dot{V}(t)=s\left(\ddot{\eta}-\ddot{\eta}_{r}\right)-\gamma \tilde{\delta} \dot{\hat{\delta}}
$$

Substituting expression for from equation (63) into (69) yields

$$
\begin{aligned}
\dot{V}(t)= & s\left[f\left(\eta_{i}, \dot{\eta}_{i}\right)-\hat{f}\left(\eta_{i}, \dot{\eta}_{i}\right)+\left(1-b \hat{b}^{-1}\right) \hat{f}\left(\eta_{i}, \dot{\eta}_{i}\right)+\left(1-b \hat{b}^{-1}\right) \hat{\delta} \hat{h}\left(\eta_{i}, \dot{\eta}_{i}\right)\right. \\
& \left.-\left(1-b \hat{b}^{-1}\right) \ddot{\eta}_{r}+\delta h\left(\eta_{i}, \dot{\eta}_{i}\right)-\hat{\delta} \hat{h}\left(\eta_{i}, \dot{\eta}_{i}\right)\right]-b \hat{b}^{-1} K|s|-\gamma \widetilde{\delta} \dot{\hat{\delta}}
\end{aligned}
$$


Assumption 3 The nonlinear function $h\left(\eta_{i}, \dot{\eta}_{i}\right)$ and $f\left(\eta_{i}, \dot{\eta}_{i}\right)$ are estimated and the estimation error is assumed to be bounded by positive known function given by (71)

$$
\begin{gathered}
\left|h\left(\eta_{i}, \dot{\eta}_{i}\right)-\hat{h}\left(\eta_{i}, \dot{\eta}_{i}\right)\right| \leqslant H\left(\eta_{i}, \dot{\eta}_{i}\right) \\
\left|f\left(\eta_{i}, \dot{\eta}_{i}\right)-\hat{f}\left(\eta_{i}, \dot{\eta}_{i}\right)\right| \leqslant F\left(\eta_{i}, \dot{\eta}_{i}\right) \\
\dot{V}(t) \leqslant s\left[f\left(\eta_{i}, \dot{\eta}_{i}\right)-\hat{f}\left(\eta_{i}, \dot{\eta}_{i}\right)+\left(1-b \hat{b}^{-1}\right) \hat{f}\left(\eta_{i}, \dot{\eta}_{i}\right)+\left(1-b \hat{b}^{-1}\right) \hat{\delta} \hat{h}\left(\eta_{i}, \dot{\eta}_{i}\right)\right. \\
\left.-\left(1-b \hat{b}^{-1}\right) \ddot{\eta}_{r}+\delta H\left(\eta_{i}, \dot{\eta}_{i}\right)\right]-b \hat{b}^{-1} K|s|-\gamma \widetilde{\theta} \dot{\hat{\theta}}+\widetilde{\delta} \hat{h}\left(\eta_{i}, \dot{\eta}_{i}\right) s
\end{gathered}
$$

$K$ should be chosen to satisfy the sliding condition

$$
\begin{gathered}
\frac{1}{2} \frac{d}{d t} s^{2} \leqslant-\alpha|s| \\
K \geqslant\left(b^{-1} \hat{b}-1\right) F\left(\eta_{i}, \dot{\eta}_{i}\right)+\alpha b^{-1} \hat{b} \\
+\left(b^{-1} \hat{b}-1\right)\left(\hat{f}\left(\eta_{i}, \dot{\eta}_{i}\right)+\hat{\delta} \hat{h}\left(\eta_{i}, \dot{\eta}_{i}\right)-\ddot{\eta}_{r}\right)+b^{-1} \hat{b} \delta_{\max } H\left(\eta_{i}, \dot{\eta}_{i}\right) .
\end{gathered}
$$

Let $b^{-1} \hat{b}=\beta$

$$
K \geqslant \beta\left(F\left(\eta_{i}, \dot{\eta}_{i}\right)+\alpha+\delta_{\max } H\right)+(\beta-1)(|\hat{u}|) .
$$

It is clear from equation (72) that $\dot{V}(t)$ is negative definite by choosing the following tuning rule

$$
\begin{gathered}
-\gamma_{l} \widetilde{\delta} \dot{\hat{\delta}}+\widetilde{\delta} \hat{h}\left(\eta_{i}, \dot{\eta}_{i}\right) s=0 \\
\dot{\hat{\delta}}=\frac{\hat{h}\left(\eta_{i}, \dot{\eta}_{i}\right) s}{\gamma_{l}} .
\end{gathered}
$$

Thus the variable added mass can be estimated adaptively using the tuning law obtained in equation (77).

\section{Results and discussion}

\subsection{Simulation setup}

The effectiveness of the proposed SMC algorithm, a simulation setup is prepared with the AUV parameters in two dimensional spaces. The identical AUVs provide the waypoints by different error vector presented in section 4 and 5 . Parameters required for simulation are the experimental data [20] and are shown in Tab. 1.

Position estimation may be defined by tracking error $e(x)$ and $e(y)$ for passivity control and $s(x)$ and $s(y)$ for SMC control. The proposed controller based on the SMC design grasping payload mass variation is validated by simulation performed using MATLAB. 
Table 6: AUV hydrodynamic parameters [20]

\begin{tabular}{|c|c|c|c|}
\hline Parameters & Values & Parameters & Values \\
\hline Mass & $2234.5 \mathrm{~kg}$ & Length & $4.215 \mathrm{~m}$ \\
\hline$X_{\dot{u}}$ & $-141.9 \mathrm{~kg}$ & $X_{u u}$ & $-35.4 \mathrm{~kg} / \mathrm{m}$ \\
\hline$Y_{\dot{v}}$ & $-1715.4 \mathrm{~kg}$ & $Y_{v v}$ & $-667.5 \mathrm{~kg} / \mathrm{m}$ \\
\hline$N_{\dot{r}}$ & $-1349 \mathrm{~kg} \cdot \mathrm{m}^{2} / \mathrm{rad}$ & $N_{r r}$ & -310 \\
\hline$N_{v v}$ & $433.8 \mathrm{~kg}$ & $X_{v r}$ & $1715.4 \mathrm{~kg} / \mathrm{rad}$ \\
\hline$Y_{u r}$ & $103.4 \mathrm{~kg} / \mathrm{rad}$ & $N_{u r}$ & $-1427 \mathrm{~kg} \cdot \mathrm{m} / \mathrm{rad}$ \\
\hline$Y_{u v}$ & $-346.76 \mathrm{~kg} / \mathrm{m}$ & $N_{u v}$ & $-686.08 \mathrm{~kg}$ \\
\hline
\end{tabular}

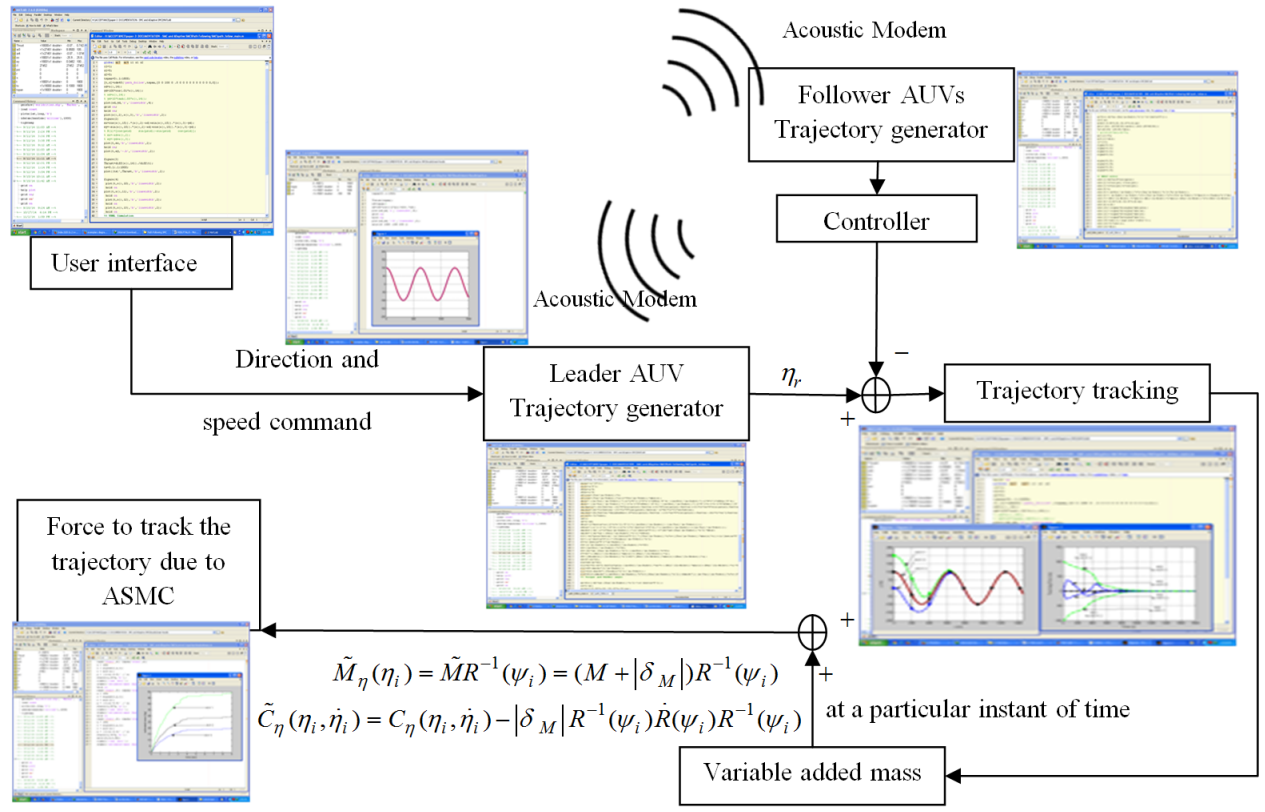

Figure 6: Exemplary diagram of formation of AUVs in MATLAB / Simulink environment.

The parameters of communication topology are used in [22]. An exemplary diagram of formation of AUVs in MATLAB/Simulink environment is as shown in Fig. 6.

$$
a_{i j}=\left\{\begin{array}{l}
1 \\
0
\end{array} \text { if AUV } j \text { in=s neighbour of AUV } i\right.
$$


where $i=1,2,3$ and the control gains are chosen as $\gamma=1, \beta=1.5$ and $\theta_{0}(0)=0$. The proposed tracking algorithm of the virtual leader is given by

$$
\begin{gathered}
\dot{\theta}_{0}=\frac{4}{\sqrt{\left(\frac{\partial x_{0}}{\partial \theta_{0}}\right)^{2}+\left(\frac{\partial y_{0}}{\partial \theta_{0}}\right)^{2}}} \\
\psi_{0}\left(\theta_{0}\right)=\arctan \left(\frac{y_{0}^{\prime}\left(\theta_{0}\right)}{x_{0}^{\prime}\left(\theta_{0}\right)}\right) .
\end{gathered}
$$

(a)

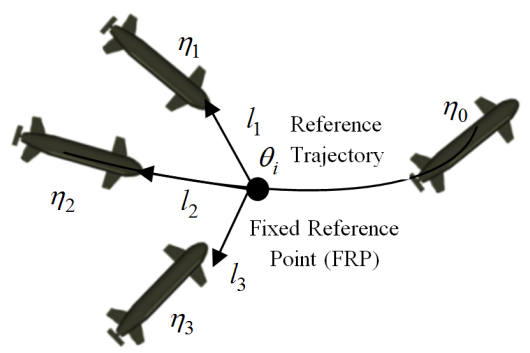

(b)

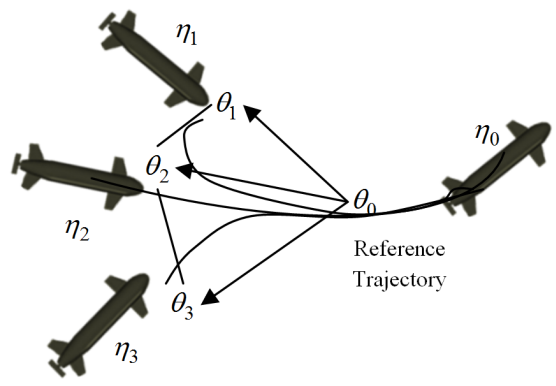

Figure 7: Formation path following virtual leader using (a) Passivity Controller (b) SMC.

Fig. 7 (a) shows that the passivity control approach to follow individual path after reaching FRP for each AUV through communication topology. The formation control using SMC following the virtual leader using same communication issue is as shown in Fig. 7 (b). The positive control gain matrix is simulated with $K_{d i}=\operatorname{diag}[2,1,1]$ and the set of designation vectors are taken as $l_{1}=[0,100,0]^{T}, l_{2}=[0,0,0]^{T}$ and $l_{3}=[0,150,0]^{T}$ [13]. The simulation parameters used in SMC are $K=\operatorname{diag}[0.001,0.001,0.001], w=$ $\operatorname{diag}[0.001,0.002,0.008], \Lambda=\operatorname{diag}[1,1,1]$. The initial positions of the three AUVs for the desired circular path are given by $\eta_{1}(0)=[50,-50,0]^{T}, \eta_{2}(0)=[0,0,0]^{T}, \eta_{3}(0)=$ $[20,-50,0]^{T}$. The desired circular path of the virtual leader is chosen as

$$
\begin{gathered}
x_{0}\left(\theta_{0}\right)=100 \sin \left(\frac{2 \pi}{625} \theta_{0}\right) \\
y_{0}\left(\theta_{0}\right)=100 \cos \left(\frac{2 \pi}{625} \theta_{0}\right) .
\end{gathered}
$$

The desired sinusoidal path of a virtual leader is given by

$$
\begin{gathered}
x_{0}\left(\theta_{0}\right)=\theta_{0} \\
y_{0}\left(\theta_{0}\right)=100 \cos \left(\frac{2 \pi}{625} \theta_{0}\right) .
\end{gathered}
$$


The initial positions are given $\eta_{1}(0)=[0,200,0]^{T}, \eta_{2}(0)=[0,0,0]^{T}, \eta_{3}(0)=$ $[0,100, \pi / 4]^{T}$ and used for simulation of the three AUVs. The desired speed of AUVs for both the trajectory is $v_{1}(0)=v_{2}(0)=v_{2}(0)=[1,0,0]^{T}$. Simulation results to illustrate the interaction between virtual leader and three AUVs using proposed techniques are shown.

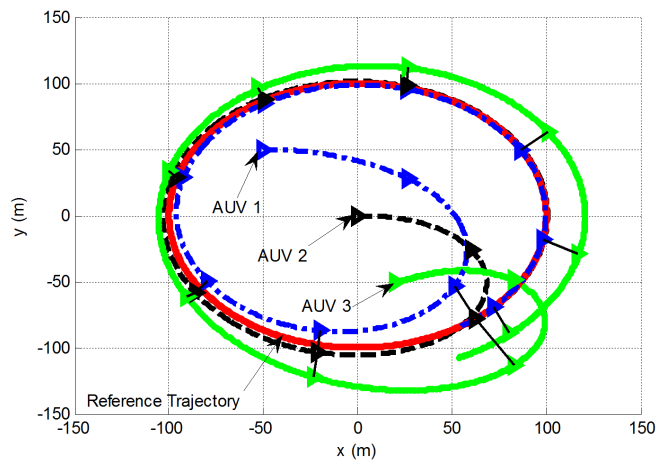

Figure 8: Formation of circular path of three AUVs following virtual leader using Passivity Controller.

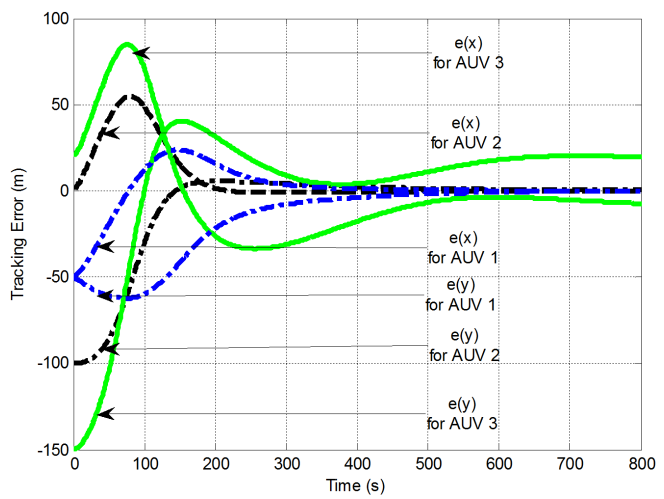

Figure 9: Tracking error of three AUVs following virtual leader using Passivity Controller for circular path.

Fig. 8 and Fig. 10 display the formation of three AUVs under circular and sinusoidal trajectory (81), (82), (83) and (84) using the passivity based control respectively. It can be observed that each AUV follows the desired trajectory by reaching its FRP and able to track the virtual reference leader due to the presence of communication link $\theta$ but unable to converge with desired trajectory.

Fig. 9 and Fig. 11 show the error generated due to positions of cooperatively coordinating AUVs. The tracking errors of the position $x$ and $y$ for follower AUV 2 converges to zero as shown in Fig. 11. Fig. 12 and Fig. 14 are represented as the SMC based trajectories of the AUVs using (81), (82), (83) and (84). Here each AUV track the virtual 


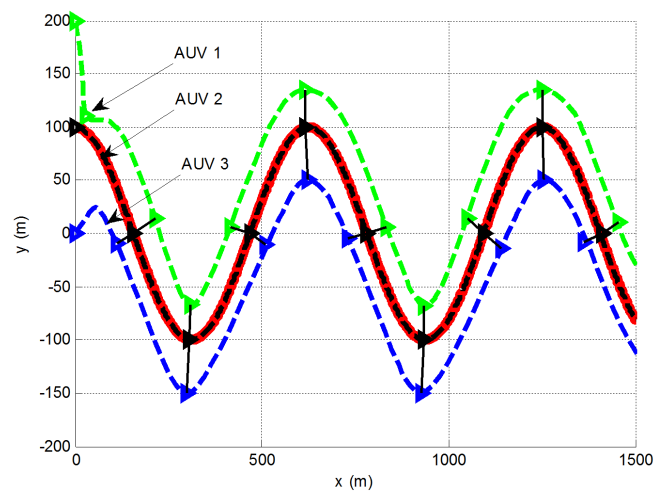

Figure 10: Formation of sinusoidal path of three AUVs following virtual leader using Passivity Controller.

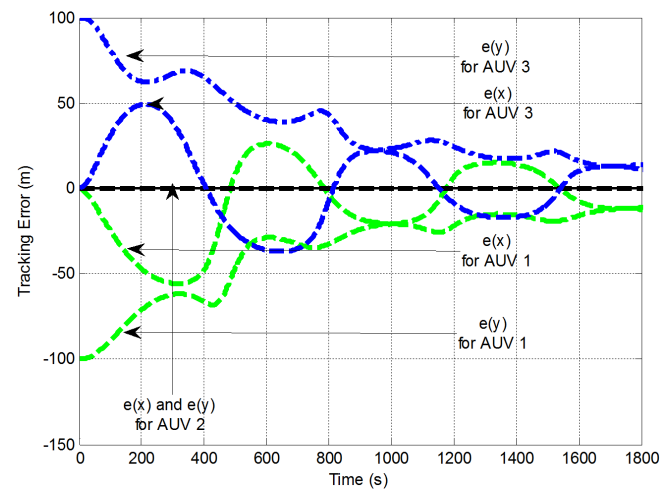

Figure 11: Tracking error of three AUVs following virtual leader using Passivity Controller for sinusoidal path.

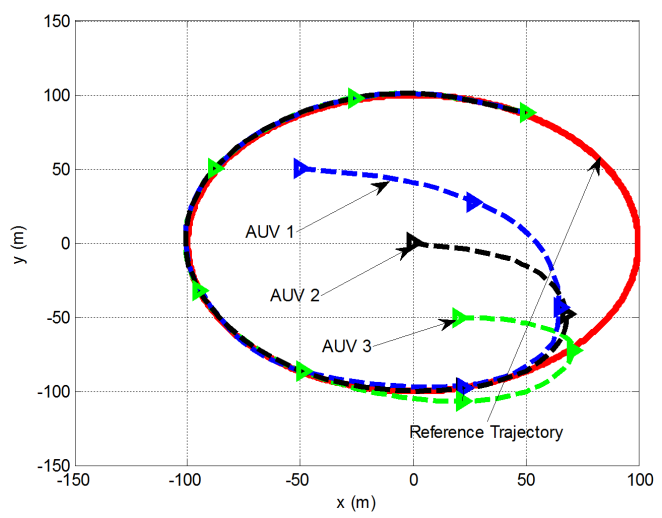

Figure 12: Formation of circular path of three AUVs following virtual leader using SMC. 


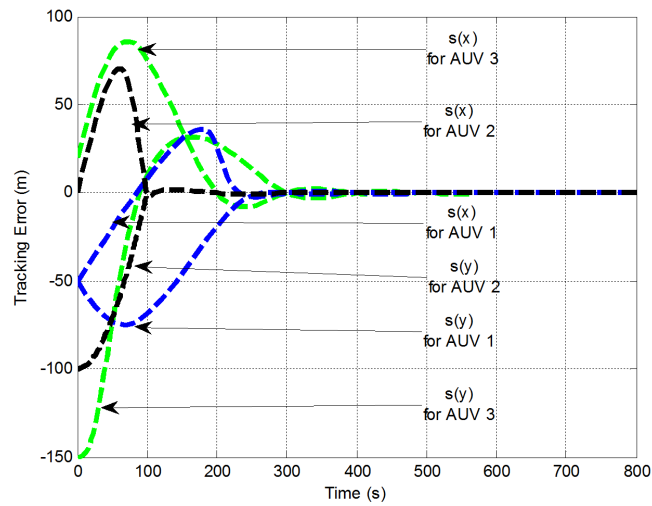

Figure 13: Tracking error of three AUVs following virtual leader using SMC for circular path.

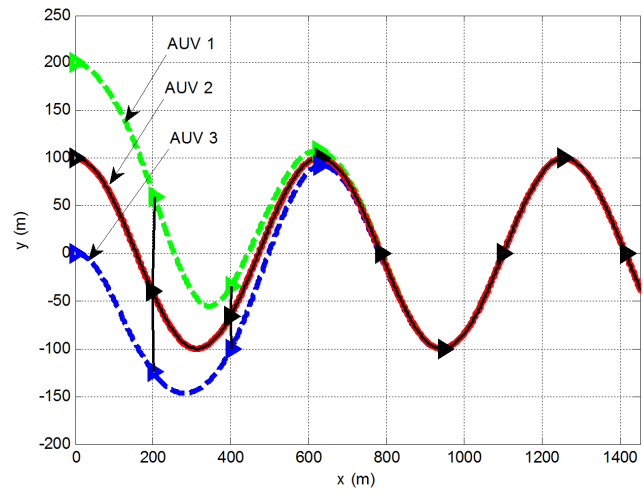

Figure 14: Formation of sinusoidal path of three AUVs following virtual leader using SMC for sinusoidal path.

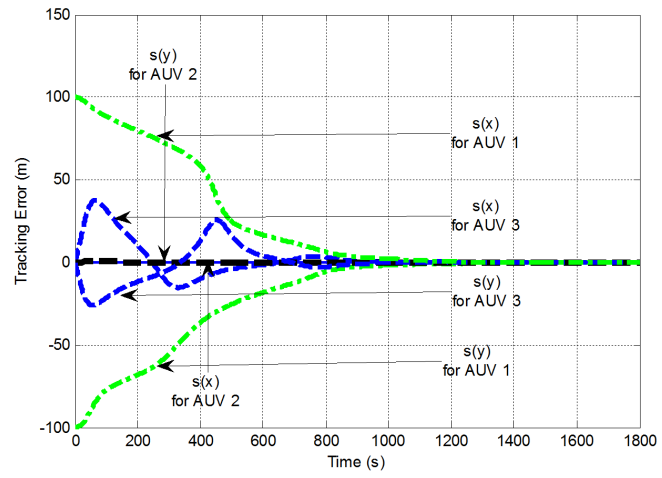

Figure 15: Tracking error of three AUVs following virtual leader using SMC. 
Table 7: Comparison of various controller techniques for tracking with respect to time using various reference trajectories

\begin{tabular}{|c|c|c|c|}
\hline Controller & Reference Trajectory & Name of AUV & Time (s) \\
\hline \multirow{6}{*}{$\begin{array}{l}\text { Passivity } \\
\text { based } \\
\text { controller }\end{array}$} & \multirow{3}{*}{ Circular } & AUV1 & $\cong 370$ \\
\hline & & AUV2 & $\cong 500$ \\
\hline & & AUV3 & $\gg 800$ \\
\hline & \multirow{3}{*}{ Sinusoidal } & AUV1 & $>1800$ \\
\hline & & AUV2 & $\cong 10$ \\
\hline & & AUV3 & $\gg 1800$ \\
\hline \multirow{6}{*}{ SMC } & \multirow{3}{*}{ Circular } & AUV1 & $\cong 230$ \\
\hline & & AUV2 & $\cong 100$ \\
\hline & & AUV3 & $\cong 430$ \\
\hline & \multirow{3}{*}{ Sinusoidal } & AUV1 & $\cong 900$ \\
\hline & & AUV2 & $\cong 5$ \\
\hline & & AUV3 & $\cong 820$ \\
\hline
\end{tabular}

reference leader due to the law of SMC and presence of communication link. Fig. 13 and Fig. 15 show the tracking error generated due to $x$ and $y$ coordinates for cooperatively coordinating AUVs. Comparison of passivity and SMC controller techniques for tracking various reference trajectories with respect to time is represented in Tab. 2.

But interesting results are obtained when a comparison is made between the proposed techniques for the same trajectory. Comparing Fig. 8 and Fig. 12, it is observed that tracking of virtual leader using SMC with same tracking consensus is better because of no designation vectors are used. It can also be outlined that all AUVs converge to the same virtual trajectory as shown in Fig. 13. Again comparing Fig. 10 and Fig. 14, it is seen that the trajectories of AUVs using SMC are better in terms of cooperative formation. Fig. 9, Fig. 11, Fig. 13 and Fig. 15 show that the time required for completely tracking virtual leader and tracking error converging to zero is less in SMC than passivity based controller. A comparison is made with same trajectory shape and initial conditions as given in Tab. 2.

The desired formation of the AUV is to have variation of the extra variable mass with a constant speed. In this research, the load varies $20 \mathrm{~kg}, 30 \mathrm{~kg}$ and $50 \mathrm{~kg}$ from AUV 1, AUV 2, and AUV 3 for circular path and $50 \mathrm{~kg}, 30 \mathrm{~kg}$ and $20 \mathrm{~kg}$ from AUV 1, AUV 2, and AUV 3 for sinusoidal path a particular instant of time respectively. Simulation results of trajectory path of the adaptive SMC for circular and sinusoidal paths are as shown in Fig.16 and 18.

Fig. 16 and Fig. 18 are the trajectory path of the formation control of the AUVs using adaptive SMC. Here for circular trajectory after 450 seconds and for sinusoidal trajectory 


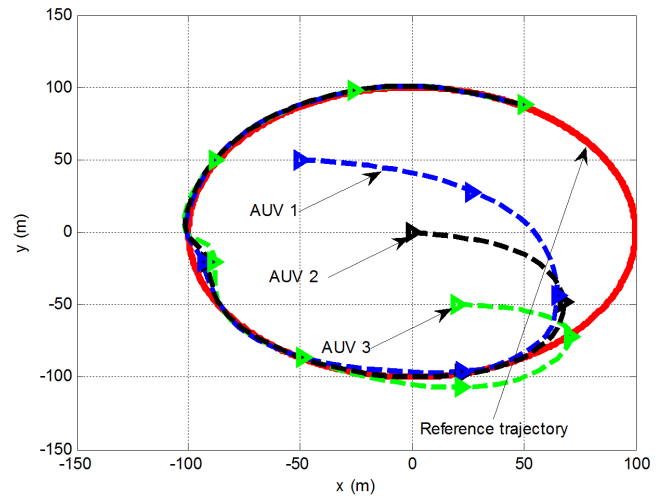

Figure 16: Formation of circular path of three AUVs following virtual leader using adaptive SMC.

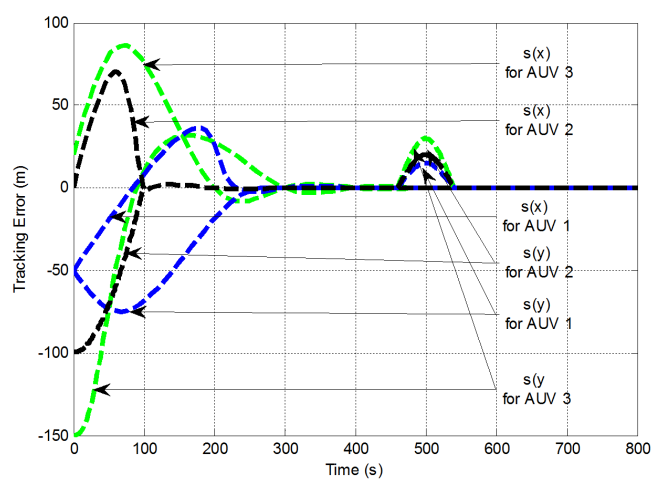

Figure 17: Tracking error of three AUVs following virtual leader using adaptive SMC for circular path.

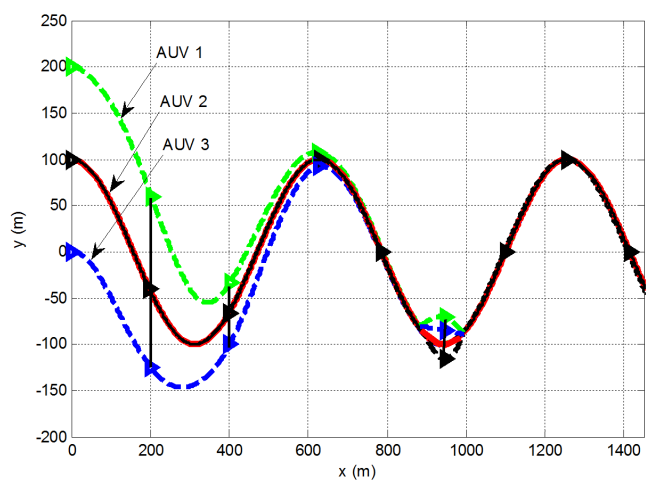

Figure 18: Formation of sinusoidal path of three AUVs following virtual leader using adaptive SMC. 


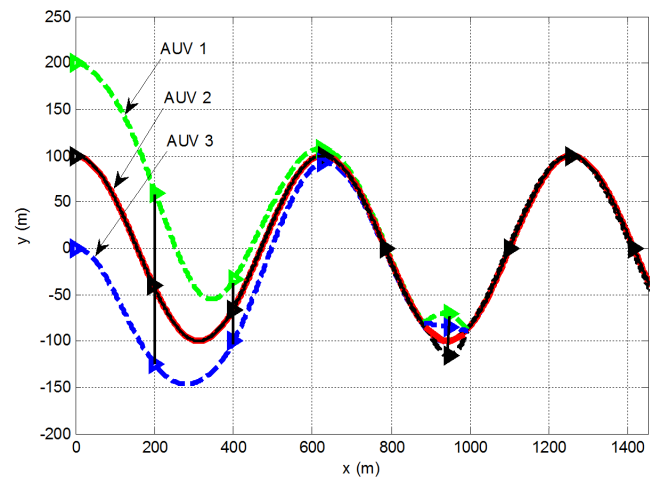

Figure 19: Tracking error of three AUVs following virtual leader using adaptive SMC for sinusoidal path.

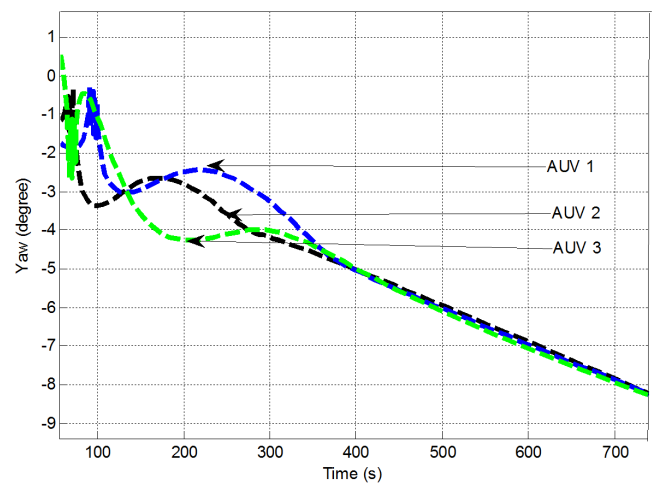

Figure 20: Euler angle of three AUVs for circular path using SMC.

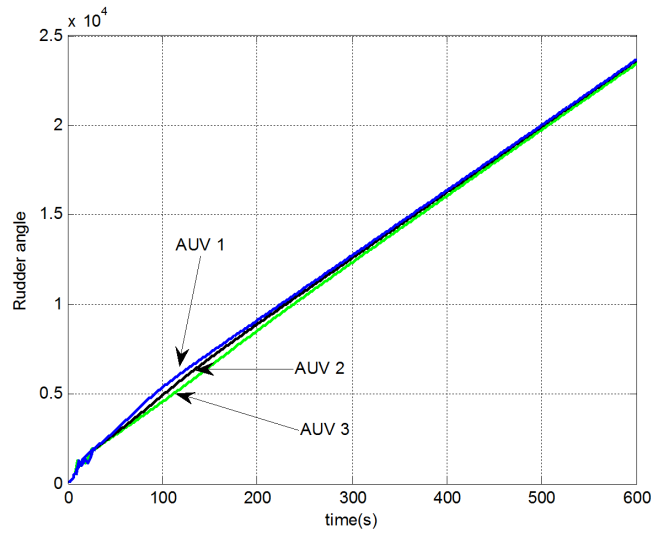

Figure 21: Rudder angle of three AUVs for circular path using SMC. 


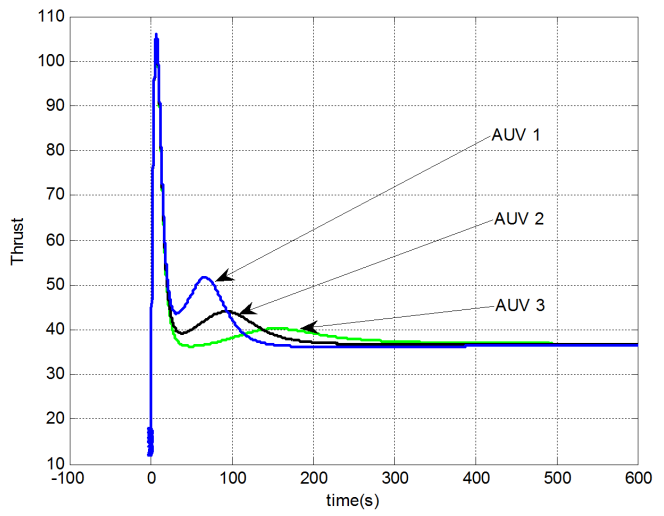

Figure 22: Thrust of three AUVs for circular path using SMC.

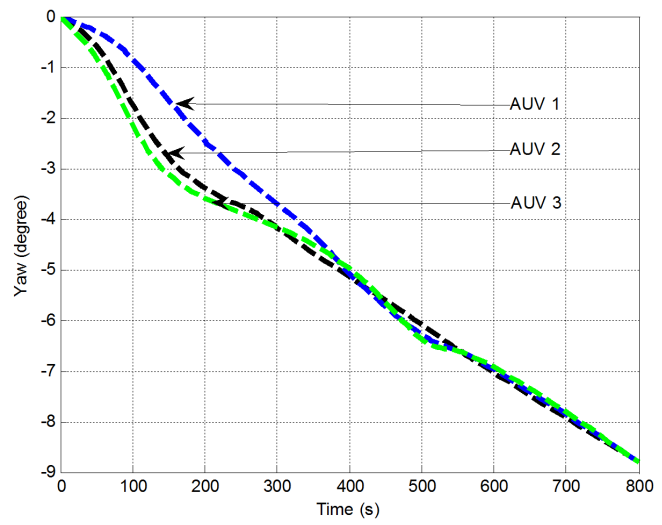

Figure 23: Euler angle of three AUVs for circular path using adaptive SMC.

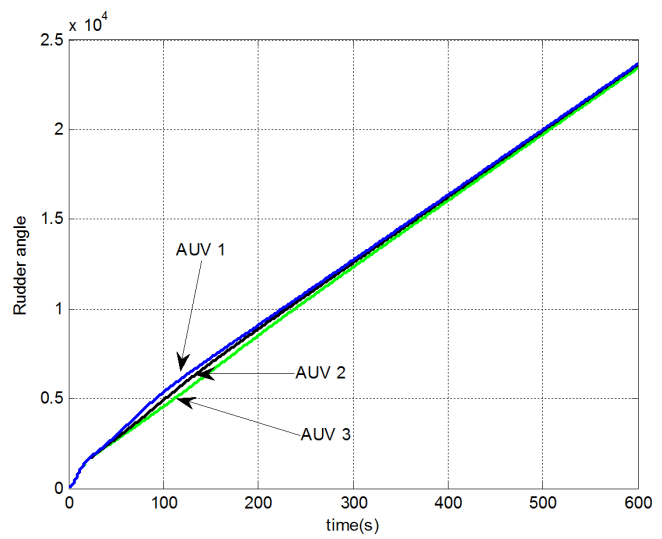

Figure 24: Rudder angle of three AUVs for circular path using adaptive SMC. 


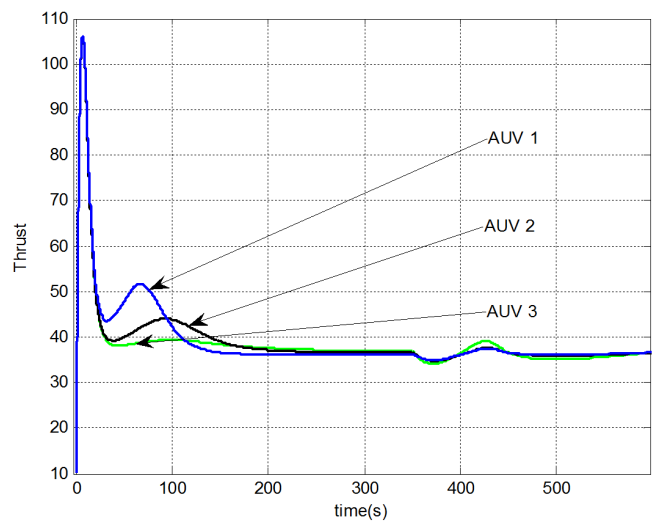

Figure 25: Thrust of three AUVs for circular path using adaptive SMC.

after 1200 seconds the load is getting varied. A small deflection can be observed but after some time period all the AUVs start to follow the same trajectories. Similarly the tracking error generated due to $\mathrm{x}$ and $\mathrm{y}$ coordinates converges to zero for cooperatively coordinating AUVs as shown in Fig. 17 and Fig. 19.

Fig. 20 to Fig. 31 show that the chattering of adaptive SMC with payload mass variation can be eliminated in comparison with SMC. Euler angles for the formation control of circular and sinusoidal are as shown in the Fig. 20, Fig. 23 and Fig. 26, Fig. 29 respectively. The control input to the SMC controller such as rudder angle and thrust are as shown in Fig. 21, Fig. 27 and Fig. 22, Fig. 28 respectively. Fig. 26 shows the robustness of the proposed SMC i.e. even under parameter uncertainty.

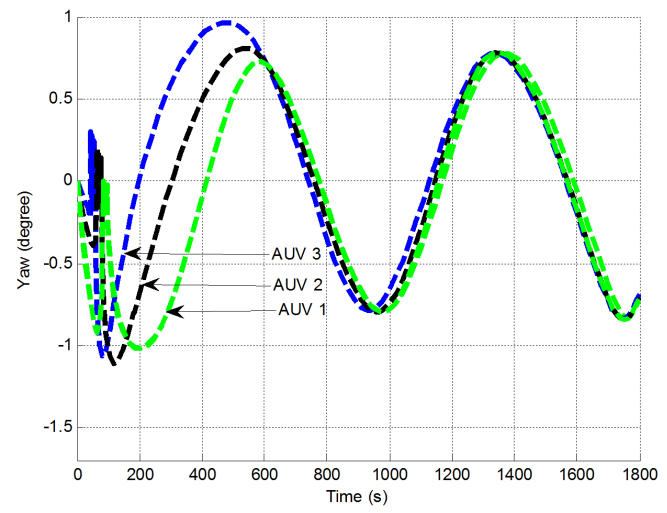

Figure 26: Euler angle of three AUVs for sinusoidal path using SMC.

The chattering function in yaw control in terms of Euler angle, rudder angle and thrust can be eliminated using adaptive SMC with payload mass variation in comparison with SMC. The rudder angle and thrust of the adaptive SMC controller for both the case of circular and sinusoidal path are as shown in Fig. 24, Fig. 25 and Fig. 30, Fig. 31. The 


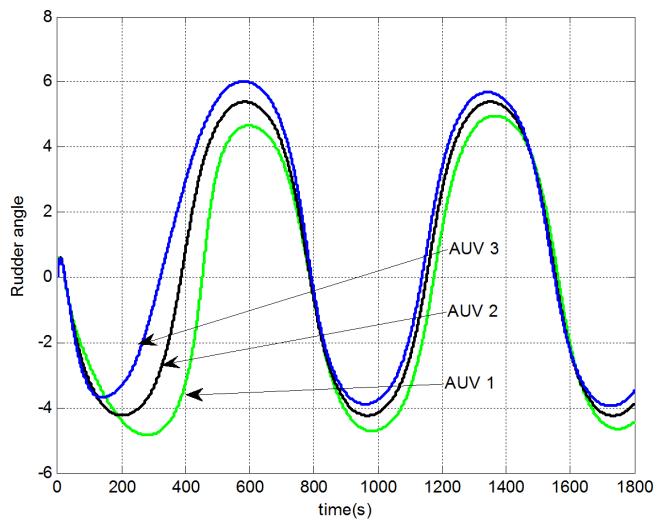

Figure 27: Rudder angle of three AUVs for sinusoidal path using SMC.

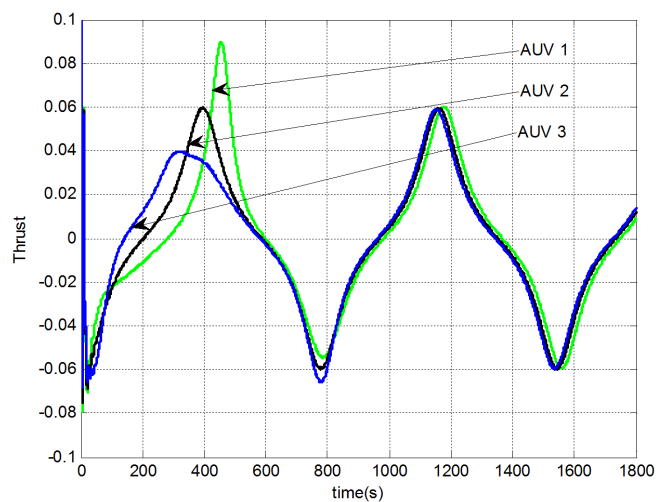

Figure 28: Thrust of three AUVs for sinusoidal path using SMC.

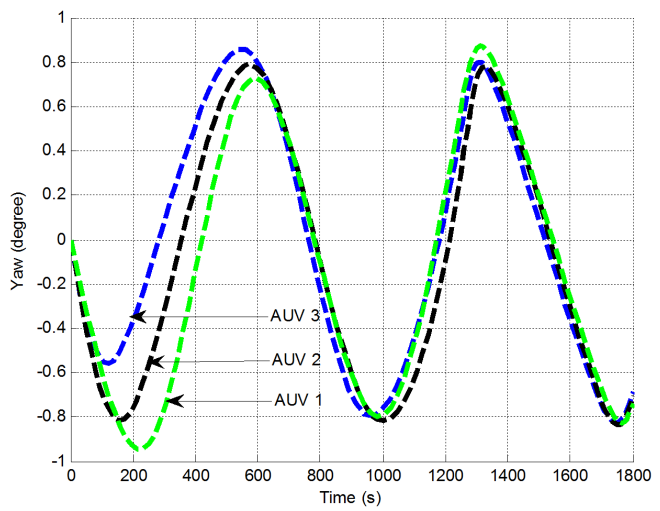

Figure 29: Euler angle of three AUVs for sinusoidal path using adaptive SMC.

controller serving the payload mass variation was similar to that of SMC with a change in rudder angle and thrust at the time of variation. 


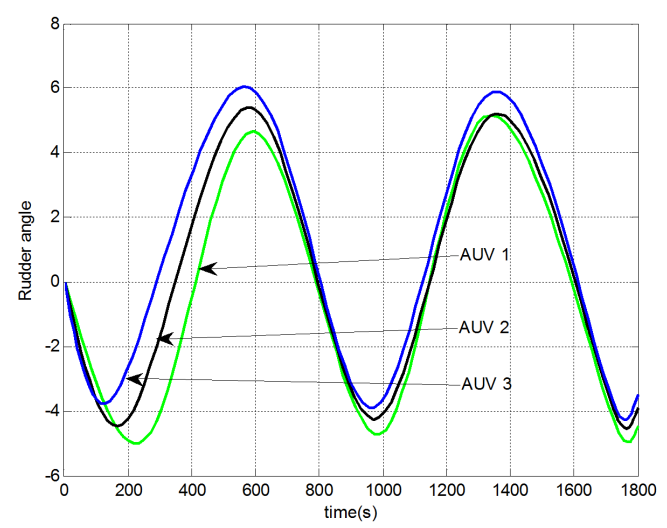

Figure 30: Rudder angle of three AUVs for sinusoidal path using adaptive SMC.

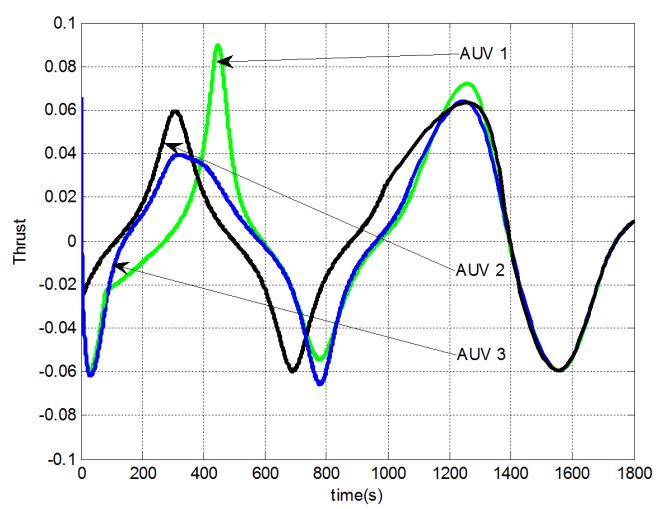

Figure 31: Thrust of three AUVs for sinusoidal path using adaptive SMC.

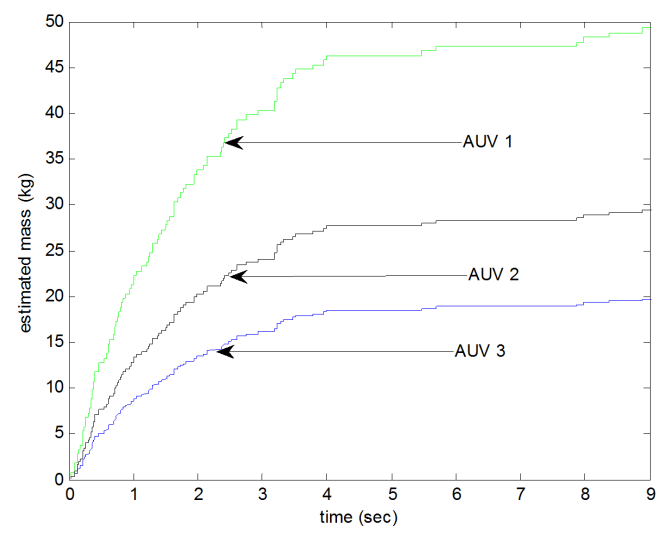

Figure 32: Estimated variable added mass for sinusoidal path using adaptive SMC. 
It can be observed from Fig. 32, the added or subtracted mass is almost estimated from AUVs in sinusoidal trajectory case.

\section{Conclusion}

In this paper we presented development of a new adaptive controller using sliding mode controller and Lyapunov theory for achieving formation control of AUVs under communication constraints. Two controllers are compared for formation of AUV trajectories under communication constraint to make all of them track the virtual leader in cooperative manner. Comparing the SMC and passivity, it is found that the former performs better based on time consumption and communication based tracking for same path. Again simulation results demonstrate that the system having adaptive SMC has strong robustness against payload mass variation by eliminating chattering phenomenon. From the obtained results it is confirmed that proposed ASMC outperforms the passivity based controller.

\section{References}

[1] J. YUH: Design and control of autonomous underwater robots: a survey. Int. J. of Control, 8(1), (2000), 7-24.

[2] A. NASIPURI and K. Li: A directionality based location discovery scheme for wireless sensor networks. Proc. of the 1st ACM Int. Workshop on Wireless Sensor Networks and Applications, New Yor, USA, (2002), 105-111.

[3] T.I. Fossen: Guidance and Control of Ocean Vehicles. Wiley, New York, 1994.

[4] E.M. Sozer, M. Stojanovic and J.G. Proakis: Underwater acoustic networks. IEEE J. of Oceanic Engineering, 25(1), (2000), 72-83.

[5] C. Godsil and G. Royle: Algebraic Graph Theory. Springer, 2001.

[6] K.D. Do: Universal controllers for stabilization and tracking of underactuated ships. System \& Control Letters, 47 (2002) 299-317.

[7] A.P. Aguiar and J.P. Hespanha: Trajectory-tracking and path-following of underactuated autonomous vehicles with parametric modeling uncertainty. IEEE Trans. on Automatic Control, 52(8), (2007), 1362-1379.

[8] W. REN and N. SoREnSEn: Distributed coordination architecture for multi-robot formation control. Int. J. of Robotics and Autonomous System, 56(4), (2008), 324333. 
[9] R. SkJETne, S. Moi and T. Fossen: Nonlinear formation control of marine craft. Proc. of the 41st IEEE Conf. on Decision and Control, bf 2 (2002), 1699-1704.

[10] H. BAI, M. ARCAK and J.T. WEN: Adaptive design for reference velocity recovery in motion coordination. System \& Control Letters, 57(8), (2008), 602-610.

[11] M. ARCAK: Passivity as a design tool for group coordination. IEEE Trans. on Automatic Control, 52(8), (2007), 1380-1390.

[12] I.F Ihle, M. ARCAK and T.I. Fossen: Passivity-based designs for synchronized path-following. Automatica, 43(9), (2007), 1508-1518.

[13] Y. WANG, W. YAN and J. Li: Passivity-based formation control of autonomous underwater vehicles. IET Control Theory \& Applications, 6(4), (2012), 518-525.

[14] W. WANG, J. Y I and D. LiU: Design of a stable sliding-mode controller for a class of second-order underactuated system. IEE Proc. of Control Theory and Application, 151(6), (2004), 691-698.

[15] J. Cheng, J. Yi and D. ZhaO: Design of a sliding mode controller for trajectory tracking problem of marine vessels. IET Control Theory \& Applications, 1(1), (2007), 233-237.

[16] R. Ghabcheloo, A. Aguiar, A. Pascoal, C. Silvestre, I. Kaminer and J. HESPANHA: Coordinated path-following control of multiple underactuated autonomous vehicles in the presence of communication failures. Proc. of the 45th IEEE Conf. on Decision and Control, (2006), 4345-4350.

[17] B. SubUDHI and S.S. GE: Sliding-mode-observer-based adaptive slip ratio control for electric and hybrid vehicles. IEEE Trans. on Intelligent Transportation Systems, 13(4), (2012), 1617-1626.

[18] P. Millan, L. Orihuela, I. Jurado and F.R. Rubio: Formation control of autonomous underwater vehicles subject to communication delays. IEEE Trans. on Control Systems Technology, 22(2), (2014), 770-777.

[19] D. Angeli and E. MoscA: Lyapunov-based switching supervisory control of nonlinear uncertain systems. IEEE Trans. on Automatic Control, 47(3), (2002), 500-505.

[20] C.J.F. SIlvestre: Multi-Objective Optimization Theory with Applications to the Integrated Design of Controllers/Plants for Autonomous Vehicles. Ph.D Thesis, Technical Institute of Lisbon, Jun. 2000.

[21] Z. Hu, C. MA, L. ZhANG and A. HAlme: Distributed formation control of autonomous underwater vehicles with impulsive information exchanges and disturbances under fixed and switching topologies. Proc. of 23rd IEEE Int. Symp. on Industrial Electronics, (2014), 99-104. 
[22] Y. CAO and W. REN: Distributed coordinated tracking with reduced interaction via a variable structure approach. IEEE Trans. on Automatic Control, 57(1), (2012), 33-48. 